\title{
Directional transport of centimeter-scale object on anisotropic microcilia surface under water
}

\author{
Yuefeng Wang ${ }^{1,6 \dagger}$, Xiaodong Chen ${ }^{2,6 \dagger}$, Kang $\operatorname{Sun}^{3 \dagger}, \mathrm{Ke} \mathrm{Li}^{4,6}$, Feilong Zhang ${ }^{5,6}$, Bing Dai ${ }^{1,6}$, Jun Shen ${ }^{4,6}$, \\ Guoqing $\mathrm{Hu}^{2,6}$ and Shutao Wang ${ }^{1,6^{*}}$
}

\begin{abstract}
Natural organisms such as cactus spines or trachea cilia have unique directional transport ability, owing to their anisotropic surface structures or asymmetric motion. However, most artificial interfacial materials are incapable of transporting macroscale object underwater. Herein, we report that anisotropic microcilia arrays, composed of cobalt fine powder and PDMS, can successfully transport the centimeterscale hydrogel underwater by periodically asymmetric stroke under alternative magnetic field. Reciprocal collective stroke of anisotropic microcilia can generate directional flow, propelling the centimeter-scale hydrogel slice forward. Accompanying computational simulation results are consistent with the directional transport behaviors observed in our experiments. This study provides a clue to design artificial anisotropic interfacial materials with capability of transporting macroscale object at low Reynolds number.
\end{abstract}

Keywords: macro-object transport, anisotropic surface, artificial micro-cilia arrays

\section{INTRODUCTION}

Anisotropic structures in nature exhibit many amazing interfacial properties, ranging from reversible adhesion of gecko feet [1], wavelength-selective reflection of butterfly wings [2], to water transport on cactus spines [3] and peristome surface of Nepenthes alata [4]. Biomimicry of these anisotropic surfaces has been emerging as an active and challenging field. Many exquisite artificial anisotropic surfaces were designed to transport milliliter droplet, for example, linear polymer grooves [5], micro ratchet glass surfaces [6], unidirectional inclined silicon nanowires [7] and nanopillars [8], tilted polydimethylsiloxane (PDMS) micropillar array [9] and nanofilm with tilted parylene nanorods [10]. Asymmetric motion of interfacial structures in organisms also exhibits distinguished transport capabilities. Taking natural cilia for instance, asymmetric stroke of their tiny and dense actuator units can provide mechanical work for directional transport of food in paramecium [11,12], mucus gel in trachea [13,14], or neuroblasts in adult brain [15]. Many responsive materials (i.e. electroactive nanowire forest [16] or membrane [17], magnetic actuated nanorods [18], micropost $[19,20]$ or sheet $[21,22]$, ion or temperature responsive hydrogel microplate [23-26] and photo-driven liquid crystal cilia [27]) were exploited to bend, twist or rotate in artificial micro actuators. However, these efforts fall into dilemma facing to directional transport of macroscale object. In air, $2 \mathrm{~cm}$-length hydrogel rod can be moved at the solid-solid interface by mechanical vibration of anisotropic surface [28] and $1 \mathrm{~cm}^{2}$ floating object can be transported at the solid-liquid interface by asymmetric bending of lightdriven polymer [29]. When under the water, only molecules, ions and micro particles can be transported directionally $[18,20,21,30,31]$. Macroscale directional transport in the liquid circumstance remains a great challenge by using an artificial interfacial material.

\footnotetext{
${ }^{1}$ CAS Key Laboratory of Bio-inspired Materials and Interfacial Science, CAS Center for Excellence in Nanoscience, Technical Institute of Physics and Chemistry, Chinese Academy of Sciences, Beijing 100190, China

2 The State Key Laboratory of Nonlinear Mechanics, Institute of Mechanics, Chinese Academy of Sciences, Beijing 100190, China

${ }^{3}$ School of Engineering, Guangdong Provincial Key Laboratory of Sensor Technology and Biomedical Instruments, Sun Yat-sen University, Guangzhou 510006, China

${ }^{4}$ Technical Institute of Physics and Chemistry, Chinese Academy of Sciences, Beijing 100190, China

${ }^{5}$ Beijing National Laboratory for Molecular Sciences (BNLMS), Key Laboratory of Green Printing, Institute of Chemistry, Chinese Academy of Sciences, Beijing 100190, China

${ }^{6}$ University of Chinese Academy of Sciences, Beijing 100049, China

These authors contributed equally to this paper.

* Corresponding author (email: stwang@mail.ipc.ac.cn)
} 
Here, for the first time, we demonstrate that anisotropic microcilia PDMS arrays mixed by cobalt nanoparticles can perform magnetically actuated transport of centimeter-scale hydrogel slice in the liquid circumstance. Transport capability of microcilia arrays can be manipulated by tuning the tilt angle, the row spacing of microcilia and the actuation frequency of magnetic field. Furthermore, experimental results and numerical analyses confirm that microcilia stroke driven by reciprocal magnetic field can effectively generate directional flow, and finally lead to transport of macroscale hydrogel slice. Our approach opens an avenue for effective transport of macroscale object at low Reynolds number by engineering anisotropic and stimuli-responsive interfacial materials.

\section{EXPERIMENTAL SECTION}

\section{Fabrication of magnetic-driven artificial microcilia arrays} with a tilt angle

The artificial microcilia arrays were fabricated through replica molding method [32,33], which is described in Fig. 1a. Firstly, polyethylene sheet (PE, low density, Alfa) was fixed on the bevel edge of right-triangle holder and then ordered conical cavities were punched by using a programmable X-Y-Z controlling platform [34]. Therefore, by tuning the holder's bevel angle, PE molds with ordered tilted conical cavities were obtained.

Secondly, cobalt nanoparticles (Aladdin) (Fig. S1) were added in PDMS prepolymer (Dow-Sylgard 184). The weight ratio of components is 10:1:5 (prepolymer:curing agent:cobalt nanoparticles). After degassing process, the mixture was squeeze coated onto the as-prepared $\mathrm{PE}$ mold. The samples were degassed again for $1 \mathrm{~h}$ and then treated by ultrasonic for $2 \mathrm{~h}$ to facilitate cobalt nanoparticles penetration. In case of the heat generated by ultrasonic process, ice was added into the water tank. Finally the homogeneous coating was cured at $80^{\circ} \mathrm{C}$ for $2 \mathrm{~h}$. After peeling off the coating carefully, we obtained the magnetic-driven, uniform and ordered microcilia arrays with different tilt angle and row spacing (Fig. S2).

\section{Macroscale hydrogel transport test for artificial microcilia arrays}

The artificial microcilia arrays, actuated by a moving magnet on the robotic arm, were capable of transporting an agar hydrogel slice at low Reynolds number. The device was exhibited in Fig. S3. The fluid used in the experiment was sodium hyaluronate (Xi'an Realin Biotechnology Co.)/phosphate buffered saline (PBS, $1 \times$,
HycloneTM) solution (150 mg sodium hyaluronate resolved in $100 \mathrm{~mL}$ PBS). The viscosity of the fluid is $13.3 \mathrm{mPa}$. This dilute biological fluid permits the potential use of artificial microcilia arrays in biomedical applications.

The macroscale object of hydrogel slices were produced as follows. Firstly, $4 \mathrm{wt} \%$ of agar powder (OXOID LP0011) solution was prepared at $80^{\circ} \mathrm{C}$. For better observation of the hydrogel slice, $5 \mathrm{wt} \%$ of titanium oxide (100 nm, rutile, Aladdin) was blended. After intensive mixing in $0.5 \mathrm{~h}$, the hot solution was poured into plastic petri dishes, which is treated by plasma washer, in order to achieve a membrane with a thickness of 150-200 $\mu \mathrm{m}$. The membrane turned hard when immersed in water. Ultimately, by tailoring the membrane with a punching bear, the slice with a diameter of $1.1 \mathrm{~cm}$ and weight of $66 \mathrm{mg}$ was obtained.

Testing process: First, the artificial microcilia arrays sample was adhered onto the bottom of the observation tank by double-side tape. The above-prepared fluid was then poured into the tank and the hydrogel slice was put onto the microcilia surface. As the robotic arm moved a magnet reciprocally in a particular frequency, the hydrogel slice was transported along with the periodical stroke of microcilia arrays. A digital camera (Cannon, 60D, Japan) or a high-speed camera (i-SPEED 3, OLYMPUS, Japan) was applied to record the movement of microcilia arrays and the displacement of hydrogel slice. Finally, the average transport velocity of hydrogel slice for each microcilia sample was counted and the experiment for each sample was repeated three times.

\section{Numerical simulations}

Numerical simulations of magnetic flux density vectors on the microcilium in the moving magnetic field and numerical simulation of flow field generated by stroking microcilia arrays were conducted by using COMSOL software. The details for the methods and analyses are discussed in Supplementary information.

\section{RESULTS AND DISCCUTION}

The magnetically actuated anisotropic microcilia arrays, composed of PDMS and nano cobalt powder, were fabricated by replica molding approach as described in Fig. 1a. The PE mold was fixed slanted and punched by a commercial needle using $\mathrm{X}-\mathrm{Y}-\mathrm{Z}$ controlling platform. Through tuning the tilt angle and position of pores on the mold, we obtained the desired microcilia arrays with different tilt angles and row spacings (Fig. S2). The diameter of microcilia ranged from 9 to $700 \mu \mathrm{m}$, and the 

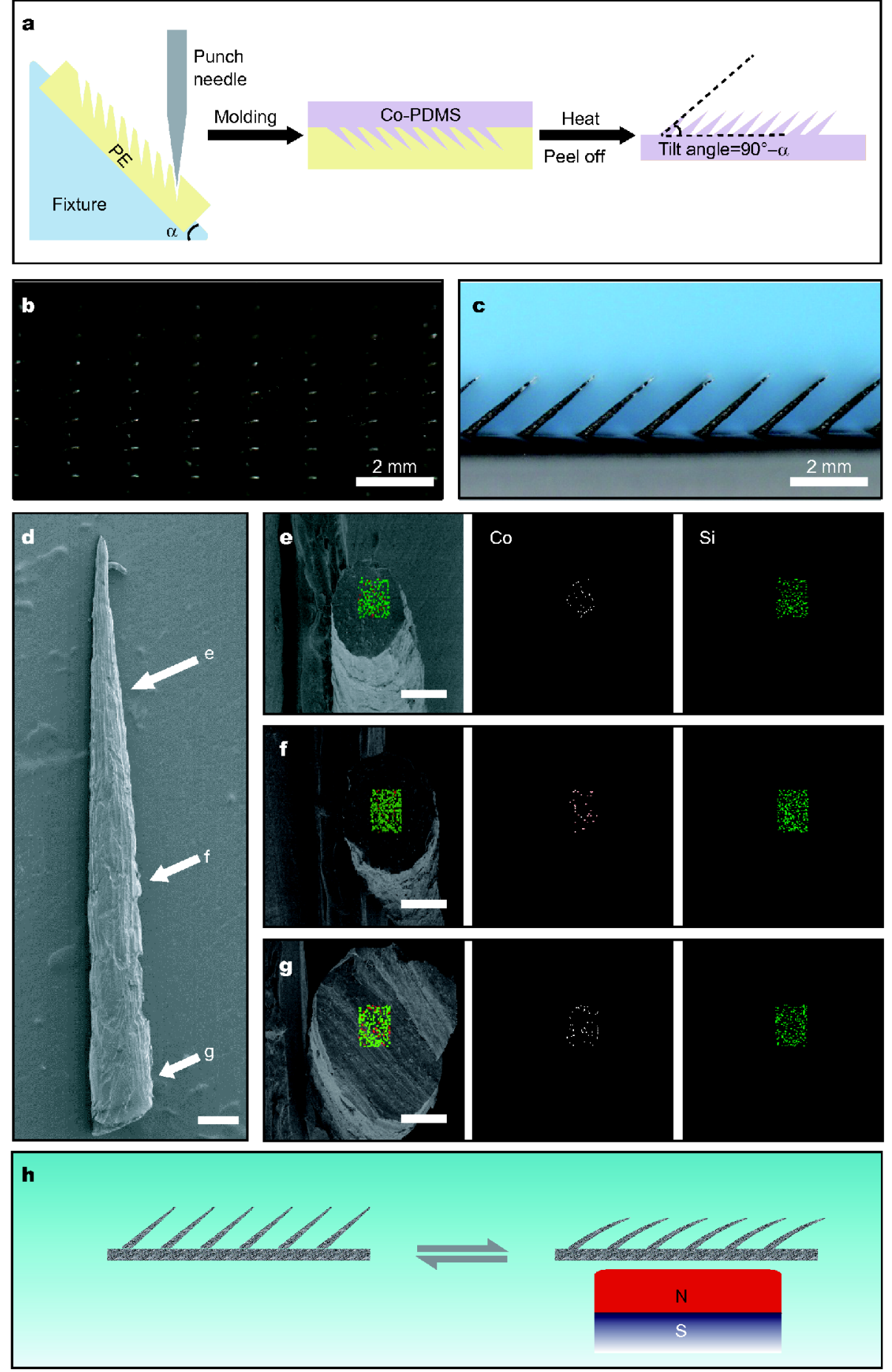

Figure 1 Fabrication and characterization of magnetic anisotropic microcilia arrays. (a) Fabrication process of the artificial microcilia arrays including preparation of anisotropic mold, curing PDMS/Co blends and peeling off replica from mold. In the process, the tilt angle of microcilia was determined by the oblique angle (a) of the mold fixture. (b) Top view and (c) side view of the artificial tilted microcilia arrays. The diameter of microcilia ranged from 9 to $700 \mu \mathrm{m}$, and the length was 2,500 $\mu \mathrm{m}$ (the cone angle was $8.5^{\circ}$ ). (d) SEM of a single cone-shape cilium. SEM and EDS of cross sections on top (e), middle ( $\mathrm{f}$ ) and bottom (g) of microcilium. (h) Schematic of the magnetic actuated tilted microcilia arrays from side view. The microcilia stroke reciprocally in the periodic changing magnetic field. Scale bars in (d)-(g) represent $100 \mu \mathrm{m}$. 

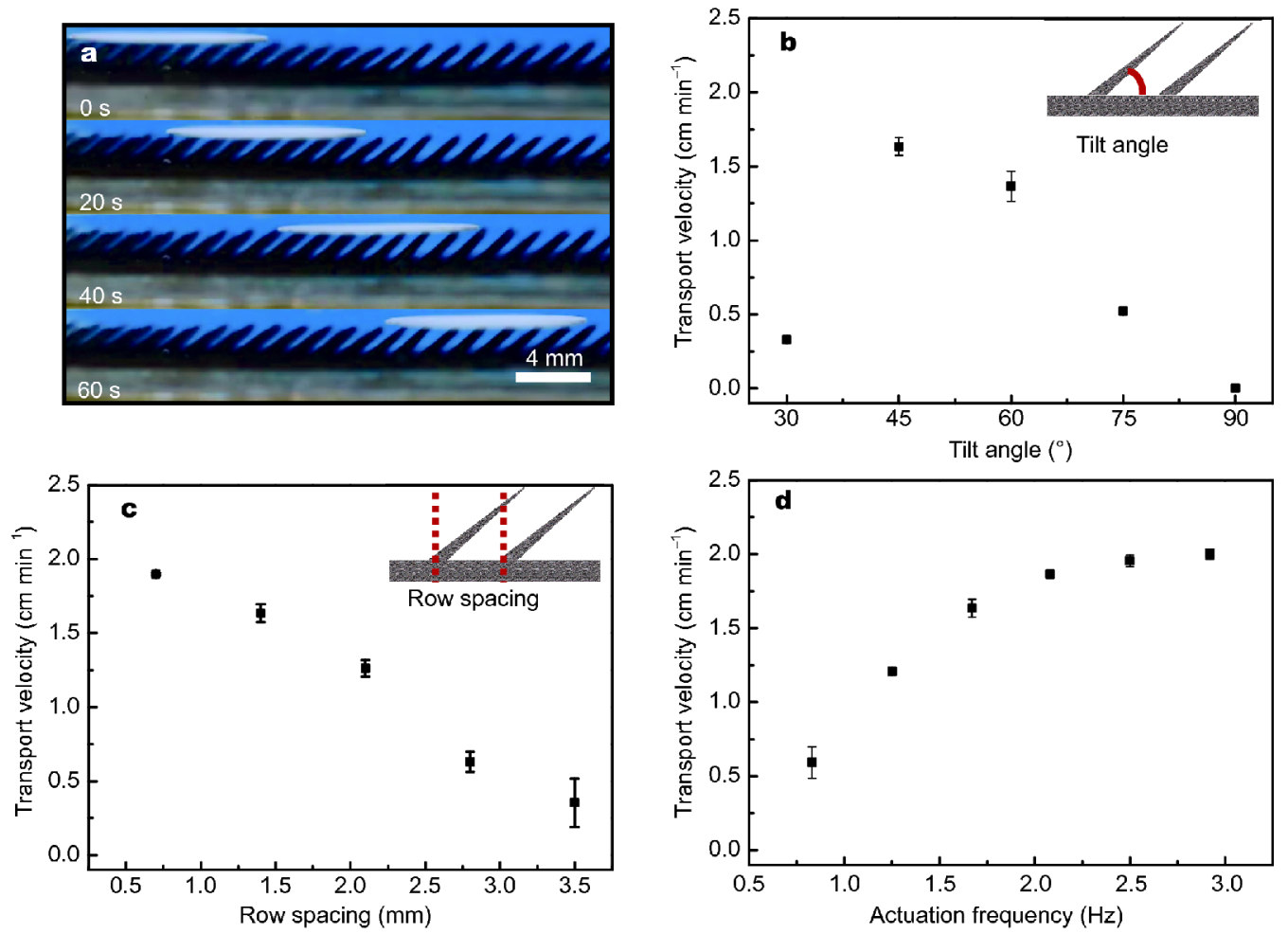

Figure 2 Transport velocity of hydrogel slice related to characteristics of microcilia arrays. (a) Centimeter-scale hydrogel was transported by stroking microcilia arrays in the liquid circumstance (see Movie S1). In this process, the tilt angle, the row spacing of microcilia arrays and the actuation frequency of magnetic field are essential factors to the transport efficiency. (b) The dependence of the transport velocity of the hydrogel slice on the tilt angle of microcilia arrays. When the tilt angle is $45^{\circ}$, the velocity achieved maximum. (c) Transport velocity of the hydrogel slice decreased monotonically with the increase of the row spacing of microcilia arrays. (d) Transport velocity of the hydrogel slice increased proportionally with the increase of the actuation frequency of magnetic field.

length was $2,500 \mu \mathrm{m}$ (the cone angle was $8.5^{\circ}$ ). In addition, as shown in Fig. $1 \mathrm{~d}-\mathrm{g}$, the cobalt element in three cross section of a microcilium was marked by a scanning electron microscope (SEM) equipped with an energydispersive X-ray spectroscope (EDS). As the section getting close to the microcilium tip, the cobalt content became lower (Table S1). This may be attributed to lower locomotion capability of cobalt nanoparticles in viscous procured PDMS. By applying an external magnetic field, the cilia array can be operated to stroke collectively (Fig. $1 \mathrm{~h})$.

We observed the dynamic transport process of agar hydrogel slice on the anisotropic artificial microcilia arrays with the tilt angle of $45^{\circ}$ in the sodium hyaluronate/ PBS solution (viscosity: $13.3 \mathrm{mPa} \mathrm{s}$ ), monitored by digital camera. The back and forth movement of magnet fixed in the robotic arm (Fig. S3) can bring periodical variation of magnetic field to actuate the anisotropic microcilia arrays. Thus, the hydrogel slice with diameter of about $1.1 \mathrm{~cm}$ was transported continuously along the tilted direction of anisotropic microcilia arrays (Fig. 2a and Movie S1).
Further, we recorded the stroking details of microcilia arrays by using a high-speed camera. Periodically changing magnetic field allows reciprocal stroke and recovery of microcilia arrays, leading to continuously step-by-step moving of hydrogel slice (Fig. S4 and Movie S2).

In order to explore the transport capability of the microcilia arrays, we explored the influence of the tilt angle, the row spacing of microcilia arrays and the actuation frequency of magnetic field on the transport velocity of hydrogel slice. Firstly, we observed the correlation of tilt angle of microcilia arrays to the transport velocity of hydrogel slice when their row spacing and the actuation frequency of magnetic field were fixed at $1.4 \mathrm{~mm}$ and $2.5 \mathrm{~Hz}$, respectively. As the tilt angle was increased from $30^{\circ}$ to $90^{\circ}$ (Fig. $2 \mathrm{~b}$ ), the transport velocity of hydrogel slice gradually increased from $0.33 \pm 0.02 \mathrm{~cm} \mathrm{~min}^{-1}$ of $30^{\circ}$ to $1.63 \pm 0.06 \mathrm{~cm} \mathrm{~min}^{-1}$ of $45^{\circ}$, a maximum transport velocity, and then decreased to $0 \pm 0.02 \mathrm{~cm} \mathrm{~min}^{-1}$ of $90^{\circ}$. Therefore, we further chose microcilia arrays with the tilt angle of $45^{\circ}$ to test the influence of the row spacing of microcilia arrays on the transport velocity of hydrogel 
slice, when the actuation frequency of magnetic field was fixed at $2.5 \mathrm{~Hz}$. As shown in Fig. 2c, when the row spacing increased from 0.7 to $3.5 \mathrm{~mm}$, the transport velocity of hydrogel slice decreased from 1.90 to $0.35 \mathrm{~cm} \mathrm{~min}^{-1}$. The result indicates that larger row spacing decreased the density of microcilia arrays, causing a reduction in the transport velocity of hydrogel slice. In Fig. 2d, when the tilt angle and the row spacing of microcilia arrays were fixed at $45^{\circ}$ and $1.4 \mathrm{~mm}$, the transport velocity of hydrogel slice increases from 0.59 to $2.00 \mathrm{~cm} \mathrm{~min}^{-1}$, with increasing actuation frequency of magnetic field from 0.83 to $2.92 \mathrm{~Hz}$. Higher actuation frequency of magnetic field causes more stroke of microcilia in a certain time, so the transport capability will increase accordingly. Therefore, we can manipulate the transport velocity of hydrogel slice within $0-2 \mathrm{~cm} \mathrm{~min}^{-1}$ by tuning the tilt angle, the row spacing of microcilia arrays and the actuation frequency of magnetic field.

In order to explore the mechanism of macroscale object transport by the microcilia arrays, we observed the transport behaviors of the $45^{\circ}$ and the vertical microcilia arrays by monitoring a blue-dyed droplet in the stroke region. Note that the blue-dyed droplet was prepared by adding solid dye powder into the solution with same components of the experimental fluid. For the $45^{\circ} \mathrm{mi}-$ crocilia arrays, the blue-dyed droplet moved upwards along the microcilia from their bottom while they were stroking. The continuous stroking of microcilia propelled blue-dyed droplet continuously moving toward the stroke direction with slight diffusion, indicating that an oriented fluid flow was generated in the horizontal direction (Fig. 3a and Movie S3). In contrast, for the vertical microcilia arrays, the blue-dyed droplet moved perpendicularly upwards along with the vertical microcilia. When propelled to the top surface of the fluid by the stroking microcilia, the dyed droplet began to separate and move symmetrically in the horizontal direction. The phenomenon indicates that the stroking by the vertical microcilia can hardly generate directional flow in the horizontal direction (Fig. $3 b$ and Movie S4).

Furthermore, we probed into the reason by numerically simulating the stroke behavior of a magnetic microcilium in the moving magnetic field. In the simulation, the magnetic flux density vectors perpendicular to the microcilium were adopted since the normal magnetic force made major contribution to its stroke and recovery. For the $45^{\circ}$ microcilium (Fig. 3c), as the magnet was moving from the right side beneath the microcilium, the magnetic flux density on the microcilium increased gradually in the left stroke direction (negative direction in the figures).
Then, the magnet crossed under the microcilium, leading to a peak value of magnetic flux density in the right stroke direction (positive direction in the figures). Finally, with the magnet moving far away from the microcilium, the magnetic flux density decreased to zero. Based on the reciprocal motion of experimental magnet, the simulated magnetic flux density curve exhibited a time-dependent periodical trend. In addition, Fig. $3 \mathrm{~d}$ also showed a combination of simulated magnetic flux density and experimental extent of microcilium bending. Although the magnetic flux density first increased in the negative direction, its relatively low value made the leftward stroke of microcilium negligible. As the positive magnetic density increased, the microcilium began to bend and its tilt angle decreased; when the magnetic flux density decreased, the microcilium recovered to its original shape due to the rubber elasticity. The asymmetric feature of magnetic flux density lead to asymmetric stroke of microcilia arrays, thus generating the directional horizontal flow of the blue-dyed liquid (Fig. 3a). For the vertical microcilium, although there is a similar trend of magnetic flux density vector, the variations of magnetic flux density in negative and positive direction were symmetric (Fig. $3 e)$. Owing to the symmetry of magnetic field, the bending and relaxation of the vertical microcilium would be in the opposite directions but in the same amplitude (Fig. 3f), causing no directional flow of blue-dyed droplet in horizontal direction (Fig. 3b).

Besides the directional flow generated by the stroking microcilia arrays, the anisotropic friction might also have influence on the transport process to the hydrogel slice $[35,36]$. We measured the density of the hydrogel slice as $1.15 \mathrm{~g} \mathrm{~cm}^{-3}$, which is a little higher than the density of experimental solution $\left(1.07 \mathrm{~g} \mathrm{~cm}^{-3}\right)$. There is friction force when the magnetic microcilia arrays contact with the hydrogel slice, which has a positive effect for the directional transport. However, as the hydrogel slice is lubricious in the solution, their friction coefficient would be very low. Therefore, we consider the main cause of directional transport of hydrogel slice is the fluid flow generated by the stroke of anisotropic microcilia arrays.

We also established a numerical model using the COMSOL software for better verifying the above-mentioned mechanism. In Fig. 4a, the numerical simulations show the velocity vectors/fields of the fluid flow generated by $45^{\circ}$ microcilia stroking. Clearly, when the microcilia stroke in the tilt direction, the horizontal flow generates on the tips of microcilia, which can form a directional propulsion force to the object on the microcilia; rather, in the recovery process, only vortex flows occurs in the gaps 

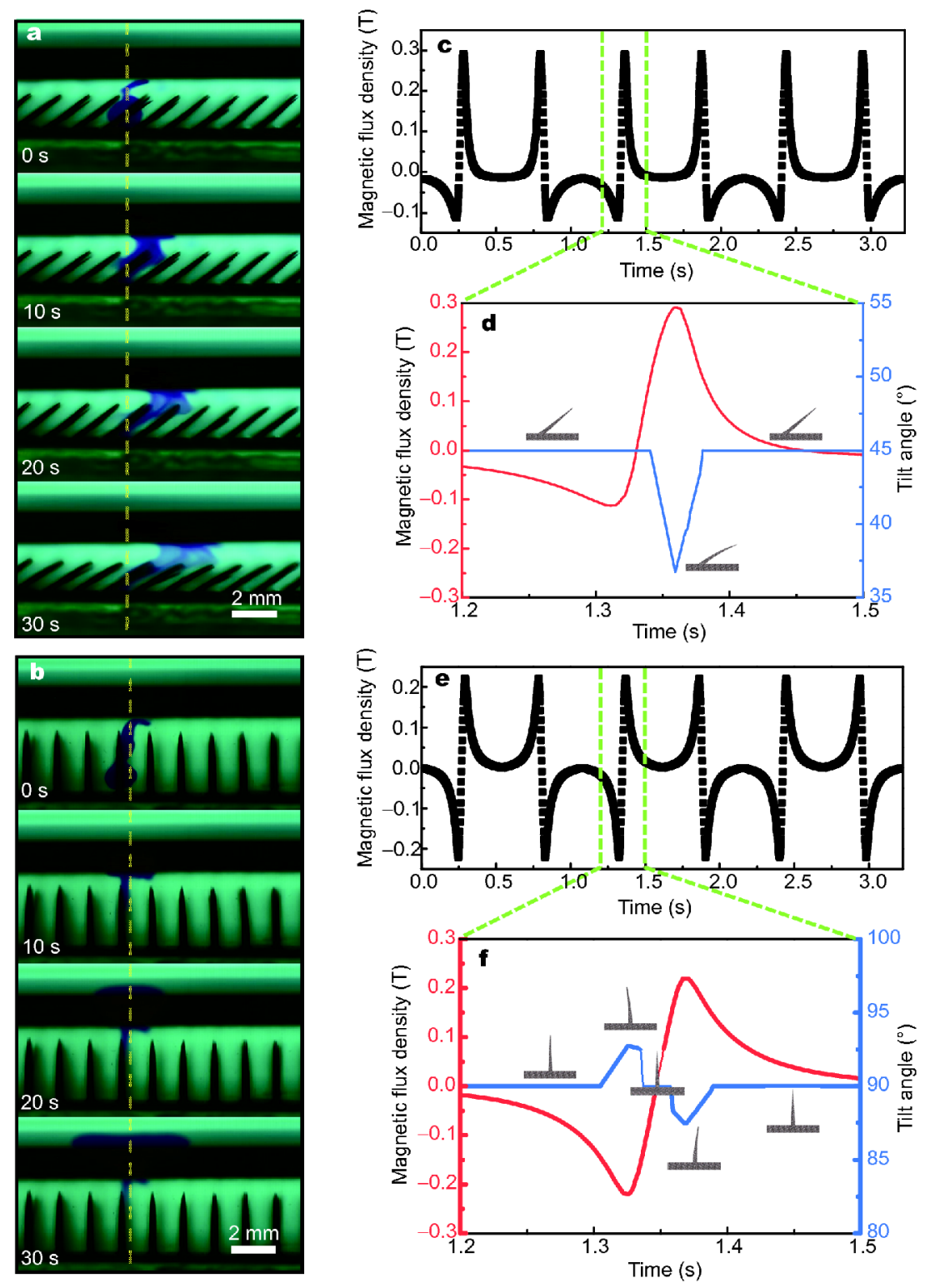

Figure 3 Generation of fluid flow by stroking microcilia arrays. A blue dyed droplet was used to monitor the fluid flow generated during the microcilia stroking. For these experiments, the row spacing of microcilia arrays and the actuation frequency of magnetic field were fixed at $1.4 \mathrm{~mm}$ and $2.5 \mathrm{~Hz}$. (a) For the microcilia arrays with the tilt angle of $45^{\circ}$, the blue dyed droplet moved toward the tilt direction along the asymmetric stroke, indicating a directional fluid flow (see Movie S3). (b) For the vertical microcilia arrays, the blue dyed droplet moved upwards and spread symmetrically on the fluid top surface, indicating that there was little directional flow generated during the symmetric stroke (see Movie S4). (c-f) Numerical simulation exhibits the magnetic flux density vectors of microcilium with tilted and vertical structures. Positive value provides the normal magnetic flux density for microcilium to stroke rightwards, and negative value provides the normal magnetic flux density for microcilium to stroke leftwards. By combining magnetic flux density vector (red curve, the left axis) with the tilt angle of microcilium tip (the blue line) during reciprocal motion of magnet, we achieved the stroke behavior for single microcilium. In (c, d), the $45^{\circ}$ microcilium showed asymmetric stroke behavior owing to asymmetric magnetic flux density in positive and negative directions. However, in (e, f), the vertical microcilium stroke symmetrically due to the balanced magnetic density in both directions.

of microcilia, which can obstruct the motion of the object. In contrast, when the microcilia are set vertical, the microcilia stroke symmetrically, and rightward and leftward velocity with symmetric distribution of magnitude were offset (Fig. 4b). Therefore, the object on the vertical microcilia can only vibrate in situ during their stroke. Ad- 


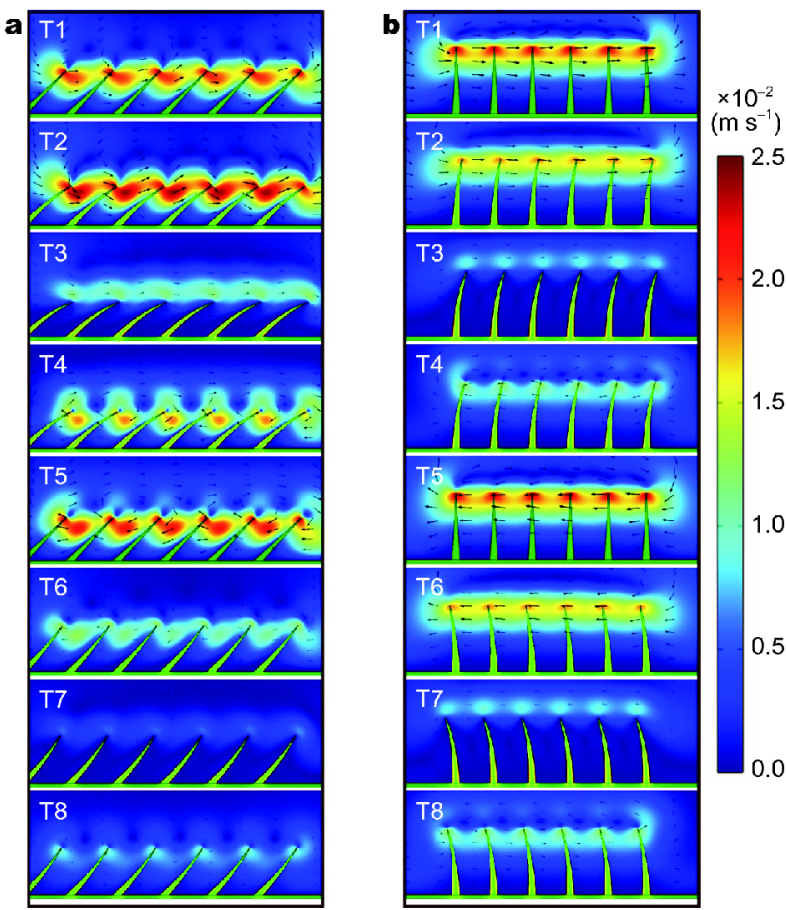

Figure 4 Numerical simulations of fluid flow by asymmetric and symmetric stroke of microcilia arrays. Movie S5 shows the velocity vectors/fields of the fluid flow around the stroking microcilia with asymmetric and symmetric structure. T1-T8 represent eight states of stroking microcilia in one cycle. T1-T4 correspond to the stroke-right process and T5-T8 correspond to the stroke-left process. (a) For $45^{\circ}$ microcilia arrays, the generated flow in T1-T4 is much larger than that in $\mathrm{T} 5-\mathrm{T} 8$, which would form propulsion force in the tilted direction. (b) For the vertical microcilia arrays, the generated flow in T1-T4 and T5T8 is completely symmetric, resulting in symmetric propulsion in the horizontal direction.

ditionally, we simulated the fluid flow generated by $45^{\circ}$ microcilia arrays with five different row spacings as in above experiments (Fig. S7a). The mean flow velocity at a horizontal plate above the numerical microcilia arrays (Fig. S7b) shows a gradual decrease with the row spacing, with the same trend of the experimental measurements of the transport velocity of hydrogel slice. The simulation results further reinforce that the major contribution to macroscale object transport is a result of the generated flow by asymmetrical stroke of microcilia arrays.

\section{CONCLUSIONS}

In summary, we have developed anisotropic magneticresponsive microcilia arrays that can transport centimeter-scale object in the liquid circumstance. Integration of magnetic nanoparticles to the anisotropic microcilia arrays endows them with asymmetric stroke in the periodical magnetic field at low Reynolds number, causing coordinated directional fluid flow and thus propelling the hydrogel forward. The magnetic-actuated microcilia arrays will show promising applications in macroscale object transport. For example, the most serious deficiency in current artificial trachea is the lack of capability of mucus gel transport [14]. Our study provides a clue to design next generation of bio-inspired interfacial materials to overcome this deficiency. Besides of magnetic actuation, other stimuli such as electricity, temperature, $\mathrm{pH}$ or light, can also be utilized to transport the macroscale object. Responding smart materials such as electroactive polymer, $\mathrm{pH}$ or temperature responsive hydrogels and light driven liquid crystal will be the candidates to design microcilia arrays. This topic of macroscale object transport is still in the infant stage and will bring a good future.

Received 1 April 2018; accepted 14 May 2018; published online 8 June 2018

1 Autumn K, Peattie AM. Mechanisms of adhesion in geckos. Integrative Comp Biol, 2002, 42: 1081-1090

2 Yoshioka S, Kinoshita S. Wavelength-selective and anisotropic light-diffusing scale on the wing of the Morpho butterfly. Proc R Soc B-Biol Sci, 2004, 271: 581-587

$3 \mathrm{Ju}$ J, Bai $\mathrm{H}$, Zheng Y, et al. A multi-structural and multi-functional integrated fog collection system in cactus. Nat Commun, 2012, 3: 1247

4 Chen $\mathrm{H}$, Zhang $\mathrm{P}$, Zhang L, et al. Continuous directional water transport on the peristome surface of Nepenthes alata. Nature, 2016, 532: 85-89

5 Xia D, He X, Jiang YB, et al. Tailoring anisotropic wetting properties on submicrometer-scale periodic grooved surfaces. Langmuir, 2010, 26: 2700-2706

6 Sandre O, Gorre-Talini L, Ajdari A, et al. Moving droplets on asymmetrically structured surfaces. Phys Rev E, 1999, 60: 29642972

7 Liu C, Ju J, Ma J, et al. Directional drop transport achieved on high-temperature anisotropic wetting surfaces. Adv Mater, 2014, 26: 6086-6091

8 Chu KH, Xiao R, Wang EN. Uni-directional liquid spreading on asymmetric nanostructured surfaces. Nat Mater, 2010, 9: 413-417

9 Cao M, Jin X, Peng Y, et al. Unidirectional wetting properties on multi-bioinspired magnetocontrollable slippery microcilia. Adv Mater, 2017, 29: 1606869

10 Malvadkar NA, Hancock MJ, Sekeroglu K, et al. An engineered anisotropic nanofilm with unidirectional wetting properties. Nat Mater, 2010, 9: 1023-1028

11 Mast SO. The food-vacuole in paramecium. Biol Bull, 1947, 92: 3172

12 Dauer DM, Ewing RM. Functional-morphology and feeding-behavior of malacoceros-indicus (polychaeta, spionidae). Bull Mar Sci, 1991, 48: 395-400

13 Fahy JV, Dickey BF. Airway mucus function and dysfunction. N Engl J Med, 2010, 363: 2233-2247

14 Button B, Cai LH, Ehre C, et al. A periciliary brush promotes the lung health by separating the mucus layer from airway epithelia. 
Science, 2012, 337: 937-941

15 Sawamoto K, Wichterle H, Gonzalez-Perez O, et al. New neurons follow the flow of cerebrospinal fluid in the adult brain. Science, 2006, 311: 629-632

16 Cheng C, Weissmüller J, Ngan AHW. Fast and reversible actuation of metallic muscles composed of nickel nanowire-forest. Adv Mater, 2016, 28: 5315-5321

17 Kim O, Shin TJ, Park MJ. Fast low-voltage electroactive actuators using nanostructured polymer electrolytes. Nat Commun, 2013, 4: 2208

18 Shields AR, Fiser BL, Evans BA, et al. Biomimetic cilia arrays generate simultaneous pumping and mixing regimes. Proc Natl Acad Sci USA, 2010, 107: 15670-15675

19 Zhou B, Xu W, Syed AA, et al. Design and fabrication of magnetically functionalized flexible micropillar arrays for rapid and controllable microfluidic mixing. Lab Chip, 2015, 15: 2125-2132

20 Wang Y, Gao Y, Wyss HM, et al. Artificial cilia fabricated using magnetic fiber drawing generate substantial fluid flow. Microfluid Nanofluid, 2015, 18: 167-174

21 Belardi J, Schorr N, Prucker O, et al. Artificial cilia: generation of magnetic actuators in microfluidic systems. Adv Funct Mater, 2011, 21: 3314-3320

22 Hu W, Lum GZ, Mastrangeli M, et al. Small-scale soft-bodied robot with multimodal locomotion. Nature, 2018, 554: 81-85

23 Sidorenko A, Krupenkin T, Taylor A, et al. Reversible switching of hydrogel-actuated nanostructures into complex micropatterns. Science, 2007, 315: 487-490

24 Zarzar LD, Kim P, Aizenberg J. Bio-inspired design of submerged hydrogel-actuated polymer microstructures operating in response to $\mathrm{pH}$. Adv Mater, 2011, 23: 1442-1446

25 He X, Aizenberg M, Kuksenok O, et al. Synthetic homeostatic materials with chemo-mechano-chemical self-regulation. Nature, 2012, 487: 214-218

26 Shastri A, McGregor LM, Liu Y, et al. An aptamer-functionalized chemomechanically modulated biomolecule catch-and-release system. Nat Chem, 2015, 7: 447-454

27 van Oosten CL, Bastiaansen CWM, Broer DJ. Printed artificial cilia from liquid-crystal network actuators modularly driven by light. Nat Mater, 2009, 8: 677-682

28 Mahadevan L, Daniel S, Chaudhury MK. Biomimetic ratcheting motion of a soft, slender, sessile gel. Proc Natl Acad Sci USA, 2004, 101: $23-26$

29 Gelebart AH, Mc Bride M, Schenning APHJ, et al. Photoresponsive fiber array: toward mimicking the collective motion of cilia for transport applications. Adv Funct Mater, 2016, 26: 5322-5327

30 Wang $\mathrm{Y}$, Gao Y, Wyss $\mathrm{H}$, et al. Out of the cleanroom, self-assembled magnetic artificial cilia. Lab Chip, 2013, 13: 3360

31 Vilfan M, Potocnik A, Kavcic B, et al. Self-assembled artificial cilia. Proc Natl Acad Sci USA, 2010, 107: 1844-1847

32 Drotlef DM, Blümler P, del Campo A. Magnetically actuated patterns for bioinspired reversible adhesion (dry and wet). Adv Mater, 2014, 26: 775-779

33 Drotlef DM, Blümler P, Papadopoulos $\mathrm{P}$, et al. Magnetically actuated micropatterns for switchable wettability. ACS Appl Mater Interfaces, 2014, 6: 8702-8707

34 Li K, Ju J, Xue Z, et al. Structured cone arrays for continuous and effective collection of micron-sized oil droplets from water. Nat Commun, 2013, 4: 2276

35 Xue L, Iturri J, Kappl M, et al. Bioinspired orientation-dependent friction. Langmuir, 2014, 30: 11175-11182

36 Jin K, Cremaldi JC, Erickson JS, et al. Biomimetic bidirectional switchable adhesive inspired by the gecko. Adv Funct Mater, 2014, 24: $574-579$

Acknowledgements This work is supported by the National Natural Science Foundation of China (21425314, 21434009, 21421061, 11402274 and 11772343), the Program for Changjiang Scholars and the TopNotch Young Talents Program of China.

Author contributions Wang Y and Wang $\mathrm{S}$ conceived the design of the research project. Wang Y performed the experiments and characterizations. Sun K and Dai B helped develop the fabrication of microcilia arrays. Li K and Shen J performed the numerical simulations of magnetic flux density vectors on the microcilium in the moving magnetic field. Chen X and Hu G developed the numerical model of stroking microcilia arrays. All authors participated in discussions and contributed to the writing of the paper. Wang $\mathrm{S}$ conceived the project and supervised the experiments. Wang Y, Chen X and Sun K contributed equally in this work.

Conflict of interest The authors declare that they have no conflict of interest.

Supplementary information Numerical simulation methods and supporting data are available in the online version of the paper. 

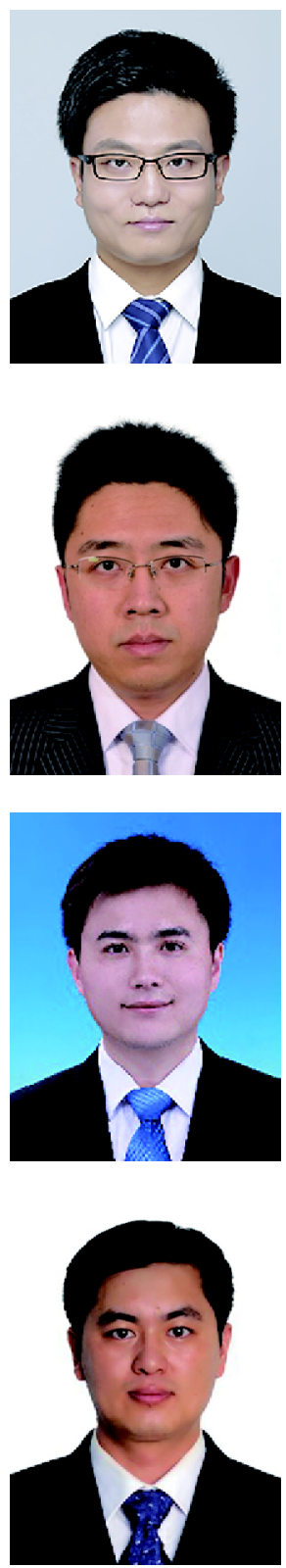

Yuefeng Wang is currently a doctoral student in the Technical Institute of Chemistry and Physics, Chinese Academy of Sciences (CAS), under the supervision of Prof. Shutao Wang. His main research focuses on bio-inspired materials.

Xiaodong Chen is currently an associate professor in the Institute of Mechanics, CAS. He received his PhD degree from the School of Astronautics, Beijing University of Aeronautics and Astronautics. He worked in the Department of aeronautics and astronautics, Georgia institute of technology as a postdoctorcal research assosiate for two years. His research interest is in the areas of micro-nano fluid mechanics and aerospace propulsion theory and engineering.

Kang Sun is currently an associate research professor at Sun Yat-sen University. He received his $\mathrm{PhD}$ degree from the National Center for Nanoscience and Technology, followed by a postdoctoral research in the Institute of Chemistry, CAS. His research interest includes bioinspired smart interface, organs on a chip and clinical test.

Shutao Wang is currently a full professor in the Technical Institute of Chemistry and Physics, CAS. He received his PhD degree from the Institute of Chemistry, CAS. His research interest focuses on the design and fabrication of bioinspired interface materials with controlled surface adhesion and nanobiointerface for theranostics.

\section{水下各向异性人造纤毛表面实现厘米尺度的块体输运}

王岳峰 ${ }^{1,6 \dagger}$, 陈晓东 ${ }^{2,6 \dagger}$, 孙康 ${ }^{3 \dagger}$, 李珂 ${ }^{4,6}$, 张飞龙 ${ }^{5,6}$, 代兵 ${ }^{1,6}$, 沈俊 ${ }^{4,6}$, 胡国庆 ${ }^{2,6}$, 王树涛 ${ }^{1,6^{*}}$

摘要 很多自然生物组织, 如猪笼草或气管纤毛, 由于具有各向异性结构或者能够不对称摆动, 而呈现出独特的定向输运功能. 但是大多仿 生材料并不能在水下对宏观物体进行输运. 在这里, 我们报道了一种具有各向异性的磁响应性纤毛结构, 能够通过其周期性的不对称摆动, 在水下驱动厘米尺度水凝胶. 在进一步的实验中, 我们观察到这种纤毛阵列的协同摆动能够产生液体的定向流动, 从而推动凝胶片向前运 动. 同时, 相关的模拟证实了实验结果. 这种能够在水下进行定向物体输运的人造纤毛结构将在微流控、生物工程等领域中发挥重要作用. 